\title{
Five-Year Follow-Up and Outcomes of Noninvasive Ventilation in Subjects With Neuromuscular Diseases
}

\author{
Mi Ri Suh MD, Won Ah Choi MD PhD, Dong Hyun Kim MD PhD, Jang Woo Lee MD, \\ Eun Young Kim MD, and Seong-Woong Kang MD PhD
}

\begin{abstract}
INTRODUCTION: The purpose of this study was to investigate the 5-year outcomes of noninvasive ventilation (NIV) application in different neuromuscular disease (NMD) groups. METHODS: We categorized 180 subjects who had initiated NIV between March 2001 and August 2009 into 4 groups and followed them for $>5 \mathrm{y}$. The NIV maintenance rate and average duration, applying time, and forced vital capacity (FVC) were investigated at the time NIV was initiated and 5 y after NIV initiation in each group. RESULTS: In subjects with amyotrophic lateral sclerosis (ALS), Duchenne muscular dystrophy (DMD), and spinal muscular atrophy (SMA)-congenital myopathy, the 5-year subjects who continued to use NIV over time were $22.5 \%, 89.4 \%$, and $91.3 \%$, respectively, and the average NIV maintenance durations were $21.53 \pm 19.26$ months, $55.22 \pm 11.47$ months, and $57.48 \pm 8.34$ months, respectively $(P<.001)$. Median daily applying time changed from $8.0 \mathrm{~h}$ to $24.0 \mathrm{~h}$ $(P<.001)$, from $8.0 \mathrm{~h}$ to $12.0 \mathrm{~h}(P<.001)$, and from $8.0 \mathrm{~h}$ to $9.0 \mathrm{~h}(P=.11)$ in subjects with ALS, DMD, and SMA-congenital myopathy, respectively. FVC decreased significantly after 5 y except in the group with combined SMA-congenital myopathy. CONCLUSIONS: NIV was tolerated long-term without significant increases in daily application time for most subjects with NMD. However, in individuals with ALS, development of severe bulbar symptoms can risk maintaining NIV. Key words: noninvasive ventilation; neuromuscular disease; duchenne muscular dystrophy; amyotrophic lateral sclerosis; spinal muscular atrophy; congenital myopathy. [Respir Care 2018;63(3):274-281. (C) 2018 Daedalus Enterprises]
\end{abstract}

\section{Introduction}

Timely application of mechanical ventilation, particularly the use of noninvasive ventilation (NIV) for respira-

Drs Suh, Choi, EY Kim, and Kang are affiliated with the Department of Rehabilitation Medicine, Gangnam Severance Hospital, Rehabilitation Institute of Neuromuscular Disease, Yonsei University College of Medicine, Seoul, South Korea. Dr DH Kim is affiliated with the Department of Rehabilitation Medicine, Kangdong Sacred Heart Hospital, Hallym University College of Medicine, Seoul, South Korea. Dr Lee is affiliated with the Department of Physical Medicine and Rehabilitation, National Health Insurance Service Ilsan Hospital, Goyang, South Korea.

A version of this article was presented by Dr Suh at AARC Open Forum 2013 held on November 17, 2013, in Anaheim, California.

Correspondence: Seong-Woong Kang MD PhD, Department of Rehabilitation Medicine and Rehabilitation Institute of Neuromuscular Disease, Gangnam Severance Hospital, Yonsei University College of Medicine, 211 Eonjuro, Gangnam-gu, Seoul, South Korea, 135-720. E-mail: kswoong@yuhs.ac.

DOI: $10.4187 /$ respcare. 05475 tory failure in patients with progressive neuromuscular diseases (NMDs), has greatly contributed to the increased lifespan and quality of life of these patients. ${ }^{1-5}$ Moreover, with the use of more diverse interfaces, access to mechanical ventilation and the scope of its application have broadened accordingly. ${ }^{6-8}$

NIV has reportedly contributed to survival without intubation in $79.2 \%$ of NMD patients who demonstrated exacerbation of respiratory symptoms..$^{9}$ In addition, it has been reported that the use of NIV has delayed the necessity for tracheostomy for months to years. ${ }^{10}$ Even for patients whose invasive ventilation weaning was difficult, a shift to continuous NIV after extubation has been suggested as a way to both remove an invasive interface and increase the survival rate. ${ }^{11}$ Similarly, NIV has helped offset the disadvantages of invasive methods and improve patients' quality of life.

However, because NMDs demonstrate various patterns of respiratory muscle weakness, the application methods and effects of NIV also vary according to the characteristics of different diseases. ${ }^{3,12,13}$ While a high percentage of 
patients with amyotrophic lateral sclerosis (ALS) eventually undergo intubation and tracheostomy due to bulbar

See the Related Editorial on Page 373

muscle dysfunction, patients with Duchenne muscular dystrophy (DMD) rarely require invasive ventilation.

Most previous long-term studies have reported NIV outcomes, such as survival rates and ventilator usage status, by setting up an end point with a specific beginning point as a basis. ${ }^{1-4}$ However, few studies have compared the condition before and after NIV in the same subjects over a long period. ${ }^{5}$

The primary aim of the study was to evaluate the number of subjects maintaining NIV for $>5 \mathrm{y}$ and the average duration of maintenance in subjects with NMDs. Second, we evaluated the variance in duration of daily NIV application at initiation and after $5 \mathrm{y}$, and we compared these differences for subjects with different NMDs. We analyzed survival rates and tracheostomy-free survival 5 y after NIV initiation, and we evaluated the variance of forced vital capacity (FVC) at NIV initiation and after $5 \mathrm{y}$ according to different types of NMD.

\section{Methods}

\section{Participants}

The subjects included NMD patients who were admitted to Gangnam Severance Hospital of Yonsei University College of Medicine, located in Seoul, South Korea, between March 2001 and August 2009. After admission, those who fulfilled the inclusion criteria received NIV. Patients whose cognitive function was too low to follow instructions and patients who did not agree to use NIV were excluded. We followed the subjects until August 2014 so that we could monitor them for at least $5 \mathrm{y}$ after NIV initiation ( \pm 6 months). The subjects were categorized into 4 groups by disease types: ALS, DMD, combined spinal muscular atrophy (SMA) and congenital myopathy (ie, these 2 diseases are relatively similar in nature ${ }^{14}$ ), and other NMDs. The other NMD group was excluded from the intergroup comparison because of the heterogeneity of disease types.

The subjects were then categorized by how they were admitted. Category A included those who were screened and found to have hypercapnia upon an out-patient regular visit without any related symptoms; Category B were those who came with acute symptoms of respiratory failure during regular out-patient visits; Category $\mathrm{C}$ were those admitted with acute symptoms of respiratory failure yet were lost to regular follow-up for an extended time and those who visited the clinic for the first time; and Category D included those who failed to wean off invasive mechanical ventilation after tracheostomy or intubation and were transferred from another

\section{QUICK LOOK}

\section{Current knowledge}

The efficacy of noninvasive ventilation (NIV) has been proven over several years. NIV has prolonged survival, decreased prolonged hospitalizations, and improved quality of life in patients with neuromuscular diseases (NMDs), including those with Duchenne muscular dystrophy (DMD), amyotrophic lateral sclerosis (ALS), spinal muscular atrophy (SMA), and congenital myopathy. There are, however, no reports showing the longterm maintenance of NIV and comparing the long-term results among different types of neuromuscular diseases.

\section{What this paper contributes to our knowledge}

The 5-y maintenance rate of NIV was high in subjects with NMD, although it showed slight differences among subgroups of ALS, DMD, combined SMA-congenital myopathy. NIV was tolerated long-term, without significant increases in daily NIV duration for most subjects with NMD. However, in individuals with ALS, development of severe bulbar symptoms can risk maintaining NIV.

hospital or other departments. The symptoms defined as being caused by respiratory failure included dyspnea at rest, morning headaches, daytime drowsiness, generalized fatigue, orthopnea, and frequent nightmares ${ }^{15}$ (Fig. 1).

\section{Study Design}

We first analyzed spot end-tidal $\mathrm{CO}_{2}$ pressure $\left(\mathrm{P}_{\mathrm{ETCO}_{2}}\right)$ and $\mathrm{S}_{\mathrm{pO}_{2}}$ and screened for subjects whose diurnal $\mathrm{P}_{\mathrm{ETCO}_{2}}$ was $\geq 40 \mathrm{~mm} \mathrm{Hg}$ and $\mathrm{S}_{\mathrm{pO}_{2}}$ was $<95 \%$ at the out-patient clinic to screen the subjects for nocturnal monitoring. For those who were intubated or had a tracheostomy, we reviewed arterial blood gas analysis or $\mathrm{P}_{\mathrm{ETCO}_{2}}$ and $\mathrm{S}_{\mathrm{PO}_{2}}$ while off mechanical ventilation. Subjects with $\mathrm{P}_{\mathrm{ETCO}_{2}}$ $\geq 40 \mathrm{~mm} \mathrm{Hg}$ and $\mathrm{S}_{\mathrm{pO}_{2}}<95 \%$, or those who had symptoms of hypoventilation, were admitted for overnight continuous monitoring. After admission, we monitored the subjects in a noninvasive and continuous manner at night, using transcutaneous oximetry $\left(\mathrm{P}_{\mathrm{tcCO}_{2}}\right) /$ capnometry (SenTec AG; Therwil, Switzerland) or end-tidal capnometry/percutaneous pulse oximetry (DASH series; GE Healthcare, Milwaukee, Wisconsin). For subjects with DMD, SMAcongenital myopathy, and other NMDs, we considered NIV when the overnight maximum $\mathrm{P}_{\mathrm{tcCO}_{2}} / \mathrm{P}_{\mathrm{ETCO}_{2}}$ was $>49 \mathrm{~mm} \mathrm{Hg}$ or the mean $\mathrm{S}_{\mathrm{pO}_{2}}$ was $<95 \%$, or when the subject had accompanying symptoms of hypoxia or hypercapnia. ${ }^{16-18}$ For subjects with ALS, we used NIV for 


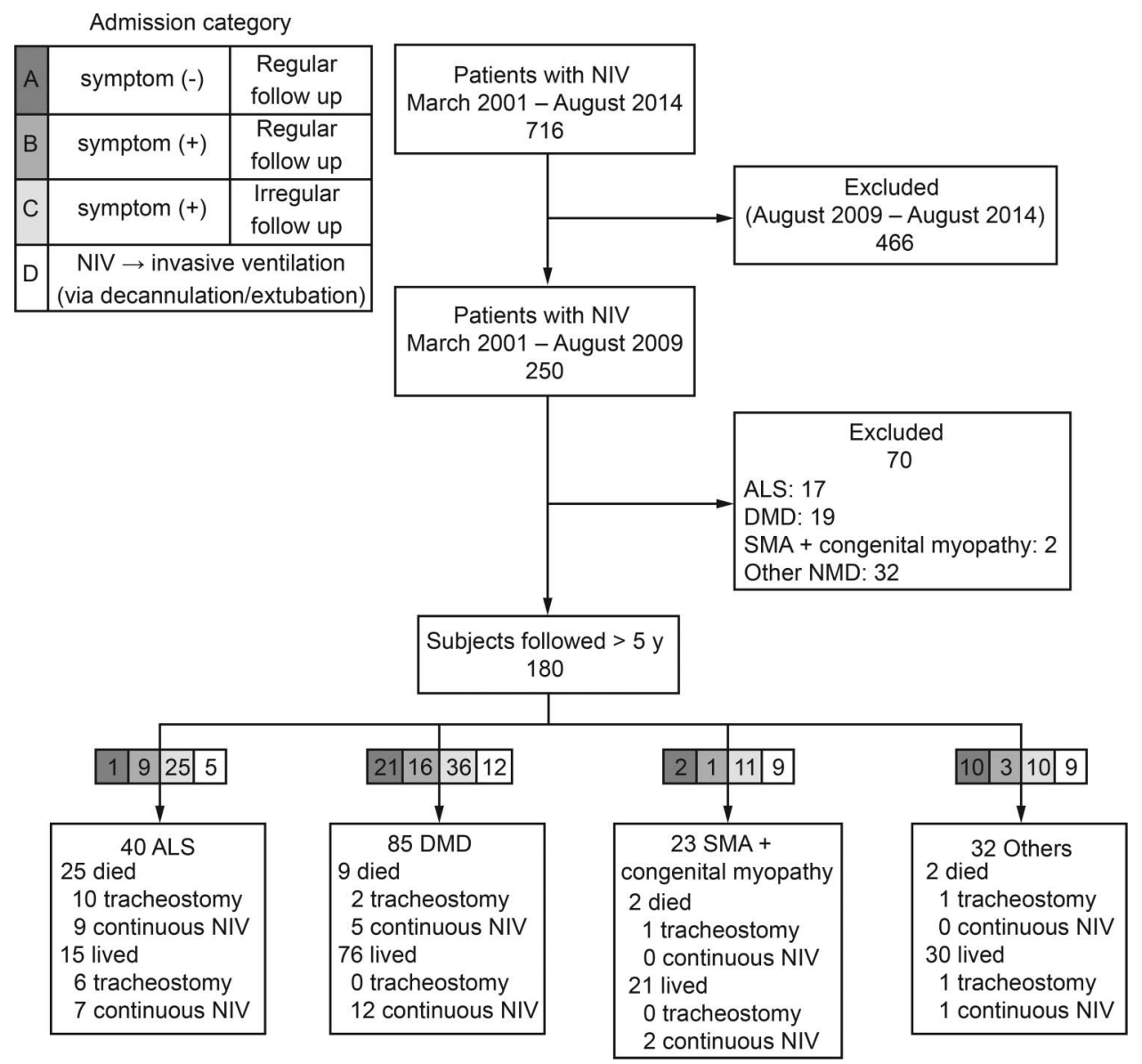

Fig. 1. Flow chart. NIV = noninvasive ventilation; continuous NIV $=$ NIV $>23 \mathrm{~h} / \mathrm{d} ; \mathrm{ALS}=$ amyotrophic lateral sclerosis; DMD $=$ Duchenne muscular dystrophy; SMA = spinal muscular atrophy; NMD = neuromuscular disease.

those with any symptoms of nocturnal hypoventilation, nocturnal monitoring showing maximal $\mathrm{P}_{\mathrm{tcCO}} / \mathrm{P}_{\mathrm{ETCO}_{2}}$ $>49 \mathrm{~mm} \mathrm{Hg}$ or nocturnal $\mathrm{S}_{\mathrm{pO}_{2}}<90 \%$ during $>5 \%$ of overnight monitoring. ${ }^{19-22} \mathrm{We}$ considered 2 or more monitoring results when the values were ambiguous, and serial values prompted the initiation of NIV. However, we did not proceed with NIV if the subject refused.

Subjects with a tracheostomy or endotracheal tube needed more processes for NIV training. For subjects with a tracheostomy, we checked the airways endoscopically and changed their tubes to a fenestrated tracheostomy tube to begin plugging the cannula while using NIV. We then checked their swallowing function to evaluate the risk of aspiration, and we trained them to cough by themselves. After their respiratory conditions were fully normalized using only NIV, subjects were decannulated. For intubated subjects, we first trained them with NIV by nasal mask without removing the endotracheal tube. When these subjects tolerated NIV and respiratory parameters were stable using this mode, endotracheal tubes were removed. Respiratory status was monitored for the first few days. Subjects who had dyspnea due to intractable airway secretions, de- saturations, or hypercapnic events after extubation underwent re-intubation.

We defined successful application of NIV as a subject whose $\mathrm{P}_{\mathrm{tcCO}_{2}} / \mathrm{P}_{\mathrm{ETCO}_{2}}$ and pulse oximetry $\mathrm{S}_{\mathrm{pO}_{2}}$ returned to normal (maximal $\mathrm{P}_{\mathrm{tcCO}_{2}} / \mathrm{P}_{\mathrm{ETCO}_{2}}<45 \mathrm{~mm} \mathrm{Hg}$ or overnight mean $\mathrm{P}_{\text {tcCO }} / \mathrm{P}_{\mathrm{ETCO}_{2}}<40 \mathrm{~mm} \mathrm{Hg}$ and nocturnal $\mathrm{S}_{\mathrm{pO}_{2}}<90 \%$ during $<5 \%$ of overnight) ${ }^{19}$ and hypoventilation symptoms were avoided using NIV overnight. We checked each subject for a need for additional daytime ventilation by continuously evaluating their respiratory status using transcutaneous oximetry/capnometry after they removed the NIV device. We measured the time until normalized $\mathrm{S}_{\mathrm{pO}_{2}}$ declined $<90 \%$ or $\mathrm{P}_{\mathrm{tcCO}_{2}} / \mathrm{P}_{\mathrm{ETCO}_{2}}$ increased $>45 \mathrm{~mm} \mathrm{Hg}$ and defined this as "ventilator-off time." Subjects received additional ventilation for $1-2 \mathrm{~h}$ after each period of ventilator-off time during the day. Subjects with ventilator-off times of $<1-30$ min were asked to apply their NIV devices continuously.

FVC was measured in both the sitting and supine positions using a spirometer (Micro Spirometer; CareFusion, Rochester, Kent, United Kingdom). At least 3 measurements were obtained for each subject, and the maximum 
Table 1. Baseline Demographics and Overall Outcomes of Subjects

\begin{tabular}{|c|c|c|c|c|c|}
\hline & $\begin{array}{c}\text { ALS } \\
(n=40)\end{array}$ & $\begin{array}{l}\text { DMD } \\
(n=85)\end{array}$ & $\begin{array}{l}\text { SMA-Congenital Myopathy } \\
\qquad(n=23)\end{array}$ & $\begin{array}{l}\text { Other* } \\
(n=32)\end{array}$ & $\begin{array}{c}\text { Total } \\
(N=180)\end{array}$ \\
\hline Male/Female, $n$ & $34 / 6$ & $85 / 0$ & $17 / 6$ & $13 / 19$ & $149 / 31$ \\
\hline Subtypes, $n$ & $\begin{array}{l}\text { Bulbar, } 3 \\
\text { Limb, } 18\end{array}$ & & $\begin{array}{c}\text { SMA type I, } 4 \\
\text { SMA type II, } 8 \\
\text { SMA type III, } 1 \\
\text { Congenital myopathy, } 10\end{array}$ & & \\
\hline Mean age $\pm S D, y$ & $57.28 \pm 11.56$ & $26.62 \pm 5.30$ & $21.70 \pm 7.93$ & $35.7 \pm 17.1$ & $34.4 \pm 16.4$ \\
\hline NIV maintenance after $5 \mathrm{y}, n(\%)$ & $9(22.5)$ & $76(89.4)$ & $21(91.3)$ & $28(87.9)$ & $134(74.4)$ \\
\hline Duration of NIV maintenance (months) & $21.53 \pm 19.26$ & $55.22 \pm 11.47$ & $57.48 \pm 8.34$ & $54.31 \pm 11.62$ & $47.86 \pm 19.35$ \\
\hline Survival after $5 \mathrm{y}, n(\%)$ & $15(37.5)$ & $76(89.4)$ & $21(91.3)$ & $30(93.8)$ & $142(78.9)$ \\
\hline Tracheostomy during 5-y period, $n(\%)$ & $16(40.0)$ & $2 \dagger(2.4)$ & $1 \dagger(4.3)$ & $2 \ddagger(6.3)$ & $21(11.7)$ \\
\hline Tracheostomy-free survival after $5 \mathrm{y}, n(\%) \S$ & $9(22.5)$ & $76(89.4)$ & $21(91.3)$ & $28(87.9)$ & $134(74.4)$ \\
\hline \multicolumn{6}{|c|}{ 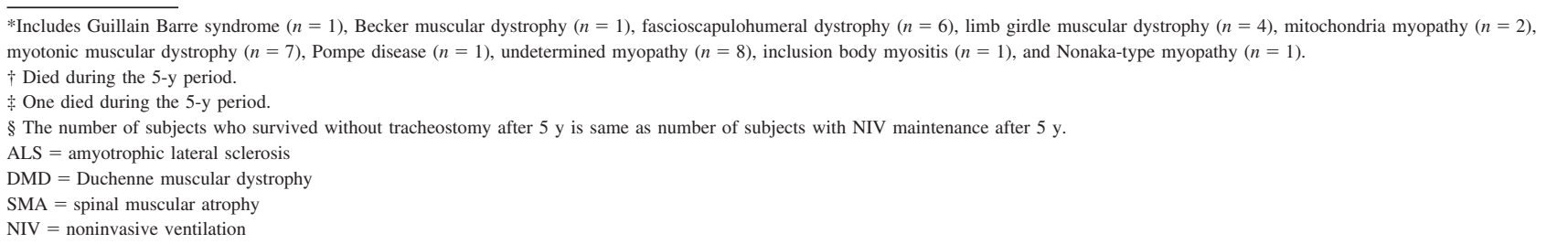 } \\
\hline
\end{tabular}

value was selected. If the FVC measurements were not high enough to be documented or could not be measured, FVC was taken to be zero in the calculation. We collected data on age, sex, intubation status, and mortality from admission and out-patient records and by telephone surveys. We also collected data regarding the NIV interface, NIV duration during the day, and FVC at initiation and at follow-up visits.

This study was approved by the Institutional Review Board of our institution (3-2013-0228). This work was financially supported by the SRC Rehabilitation Hospital 2014 Research Assistance Program of the Pulmonary Rehabilitation Center of Gangnam Severance Hospital.

\section{Statistical Analysis}

For statistical analysis, SPSS version 22.0 software (SPSS, Chicago, Illinois) was used. The Kruskal-Wallis test was used to analyze overall differences among the subgroups in NIV duration at initiation and after $5 \mathrm{y}$, NIV maintenance period, and FVC at initiation and after $5 \mathrm{y}$. The Mann-Whitney $U$ test was performed to analyze differences of median NIV duration at initiation and after 5 y between subgroups (ALS vs DMD, ALS vs SMA-congenital myopathy, and DMD vs SMA-congenital myopathy). The Wilcoxon rank-sum test was performed to compare the median NIV duration and mean FVC at initiation and after 5 y. Statistical significance was set as $P<.05$.

\section{Results}

\section{General Characteristics of Subjects}

Among the 250 patients who used NIV from March 2001 to August 2009, the survival status of 180 subjects could be verified. The remaining 70 patients were lost during follow-up and were excluded from the final analysis.

The ALS group had 40 subjects, the DMD group had 85 subjects, the SMA-congenital myopathy group had 23 subjects, and the other NMD group had 32 subjects (Guillain Barre syndrome [ $n=1]$, Becker muscular dystrophy $[n=1]$, fascioscapulohumeral dystrophy $[n=6]$, limb girdle muscular dystrophy $[n=4]$, mitochondria myopathy $[n=2]$, myotonic muscular dystrophy $[n=7]$, Pompe disease $[n=1]$, undetermined myopathy $[n=8]$, inclusion body myositis $[n=1]$, and Nonaka-type myopathy $[n=1])$. Of the 180 subjects, 149 were male and 31 were female. The mean age of the subjects was $34.4 \pm 16.4 \mathrm{y}$ (Table 1).

\section{Course to NIV Application}

Of the 180 subjects, 34 were admitted asymptomatically through screening in the out-patient clinic (Category A), 29 were admitted after developing symptoms during regular follow-up (Category B), 82 had never been under regular observation but were admitted after developing acute symptoms (Category C), and 35 were admitted with 
Table 2. NIV Duration Variance After $5 \mathrm{Y}$

\begin{tabular}{|c|c|c|c|c|c|c|}
\hline & \multirow{2}{*}{$\begin{array}{c}\text { ALS } \\
(n=40)\end{array}$} & \multirow{2}{*}{$\begin{array}{l}\text { DMD } \\
(n=85)\end{array}$} & \multirow{2}{*}{$\begin{array}{l}\text { SMA-Congenital } \\
\text { Myopathy } \\
(n=23)\end{array}$} & \multicolumn{3}{|c|}{ Post Hoc Analysis $(P)$} \\
\hline & & & & ALS vs. DMD & $\begin{array}{c}\text { ALS vs. SMA- } \\
\text { Congenital Myopathy }\end{array}$ & $\begin{array}{l}\text { DMD vs. SMA- } \\
\text { Congenital Myopathy }\end{array}$ \\
\hline $\begin{array}{r}\text { NIV duration at } \\
\text { initiation, } \mathrm{h} / \mathrm{d}\end{array}$ & $8.0(8.0-19.5)$ & $8.0(8.0-9.0)$ & $8.0(8.0-8.0)$ & .14 & .75 & .20 \\
\hline $\begin{array}{l}\text { NIV duration } \\
\text { after } 5 \mathrm{y}, \mathrm{h} / \mathrm{d}\end{array}$ & $24.0(24.0-24.0)$ & $12.0(9.5-22.0)$ & $9.0(8.0-11.0)$ & $<.001 \dagger$ & $<.001 \dagger$ & $.002 \dagger$ \\
\hline$P$ & $<.001^{*}$ & $<.001 *$ & .11 & NA & NA & NA \\
\hline \multicolumn{7}{|c|}{$\begin{array}{l}\text { Values are median (interquartile range). } \\
* \text { NIV duration at initiation and after } 5 \mathrm{y} . \\
\dagger \text { intergroup comparison of NIV duration. } \\
\text { ALS = amyotrophic lateral sclerosis } \\
\text { DMD = Duchenne muscular dystrophy } \\
\text { SMA = spinal muscular atrophy } \\
\text { NIV = noninvasive ventilation } \\
\text { NA = not applicable }\end{array}$} \\
\hline
\end{tabular}

a tracheostomy tube or endotracheal tube (Category D). Although the highest proportion of subjects was admitted by Category $\mathrm{C}(45.6 \%)$, the total admission from regular visits was 63 subjects, which accounted for as much as $35.0 \%$ (Categories A and B). Category D (ie, 35 subjects referred from another hospital with a tracheostomy tube or endotracheal tube) consisted of 5 subjects from the ALS group (5 extubated, and none decannulated), 12 from the DMD group (8 extubated, and 4 decannulated), 9 from the SMA-congenital myopathy group (3 extubated, and 6 decannulated), and 9 from the other NMD group (5 extubated, and 4 decannulated; Fig. 1).

\section{Maintenance of NIV and Daily Duration Variance}

The subjects were followed for 5 y ( \pm 6 months) from the initiation of NIV. Subjects who died were followed until the time of death. The mean follow-up period was $4.4 \pm 1.6 \mathrm{y}$. On follow-up observation, 134 of the 180 subjects were continuously using NIV (74\%). In each group, the 5-year subjects who continued to use NIV over time were $23 \%, 89 \%$, and $91 \%$ and the average NIV maintenance duration was $21.5 \pm 19.3$ months, $55.2 \pm 11.5$ months, and $57.5 \pm 8.3$ months for ALS, DMD, and SMA-congenital myopathy, respectively $(P<.001)$.

Median daily NIV duration changed from $8.0 \mathrm{~h}$ to $24.0 \mathrm{~h}$ $(P<.001), 8.0 \mathrm{~h}$ to $12.0 \mathrm{~h}(P<.001)$, and $8.0 \mathrm{~h}$ to $9.0 \mathrm{~h}$ $(P=.11)$ in ALS, DMD, and SMA-congenital myopathy, respectively. While NIV duration did not change significantly in the SMA-congenital myopathy group, it changed significantly after $5 \mathrm{y}$ in the ALS group and the DMD group. Comparing subgroups (eg, ALS vs DMD, ALS vs SMA-congenital myopathy, and DMD vs SMA-congenital myopathy), daily duration at NIV initiation showed no significant differences $(P=.14, P=.75$, and $P=.20$, respectively). However, daily NIV duration after 5 y showed significant differences between all comparisons $(P<.001$, $P<.001$, and $P=.002$, respectively) (Table 2 ).

\section{Survival After NIV Use}

Among the 180 subjects, 142 survived (79\%) through the 5-y observation period: 15 from the ALS group (15/40, $38 \%), 76$ from the DMD group (76/85, 89\%), 21 from the SMA-congenital myopathy group $(21 / 23,91 \%)$, and 30 from the other NMD group (30/32, 94\%). Tracheostomyfree survival, which is same as NIV maintenance rate after $5 \mathrm{y}$, was $22.5 \%, 89.4 \%, 91.3 \%$, and $87.9 \%$ in the ALS group, DMD group, SMA-congenital myopathy group, and other NMD group, respectively (Table 1).

Most of the NMD subjects received tracheostomy during episodes of acute deterioration except for 8 subjects with ALS. A total of 21 subjects (12\%) underwent tracheostomy (16 in the ALS group, 2 in the DMD group, 1 in the SMA-congenital myopathy group, and 2 in the other NMD group). Eight of the subjects with ALS underwent tracheostomy due to acute deterioration, and the other 8 underwent tracheostomy tube due to progressive bulbar dysfunction.

\section{FVC Evaluation}

We compared FVC measurements at NIV initiation and after $5 \mathrm{y}$. Mean FVC while in the sitting position dropped significantly from $1,108.1$ to $25.7 \mathrm{~mL}(P<.001)$ in the ALS group and from 644.9 to $323.2 \mathrm{~mL}(P<.001)$ in the DMD group, whereas it increased from 586.2 to $692.5 \mathrm{~mL}$ $(P=.20)$ the in SMA-congenital myopathy group. FVC in the supine position showed a similar pattern of change. While median FVC in the supine position decreased sig- 
Table 3. Forced Vital Capacity Variance After 5 Years

\begin{tabular}{|c|c|c|c|c|c|c|}
\hline & $\begin{array}{l}\text { Initial FVC } \\
\text { Sitting (mL) }\end{array}$ & $\begin{array}{l}\text { FVC Sitting After } \\
5 \text { Years }(\mathrm{mL})\end{array}$ & $P$ & $\begin{array}{c}\text { Initial FVC } \\
\text { Supine (mL) }\end{array}$ & $\begin{array}{c}\text { FVC Supine After } \\
5 \text { Years }(\mathrm{mL})\end{array}$ & $P$ \\
\hline ALS & $1,108.1 \pm 15.2$ & $25.7 \pm 83.4$ & $<.001 *$ & $1,112.9 \pm 17.1$ & $29.5 \pm 94.1$ & $<.001 *$ \\
\hline DMD & $644.9 \pm 14.8$ & $323.2 \pm 383.0$ & $<.001 *$ & $630.1 \pm 14.1$ & $310.7 \pm 362.9$ & $<.001 *$ \\
\hline SMA-congenital myopathy & $586.2 \pm 443.7$ & $692.5 \pm 381.8$ & .20 & $542.3 \pm 11.2$ & $614.6 \pm 359.4$ & .29 \\
\hline $\begin{array}{l}\text { Values are mean } \pm \text { SD. } \\
* \text { Initial FVC and FVC after } 5 \mathrm{y} . \\
\text { ALS = amyotrophic lateral sclerosis } \\
\text { DMD = Duchenne muscular dystrophy } \\
\text { FVC = Forced Vital Capacity } \\
\text { SMA = spinal muscular atrophy }\end{array}$ & & & & & & \\
\hline
\end{tabular}

nificantly from $1,112.9$ to $29.5 \mathrm{~mL}(P<.001)$ in the ALS group and from 630.1 to $310.7 \mathrm{~mL}(P<.001)$ in the DMD group, it increased from 542.3 to $614.6 \mathrm{~mL}(P=.29)$ in the SMA-congenital myopathy group (Table 3 ). KruskalWallis analysis showed significant differences among the subgroups for FVC in the sitting position at NIV initiation $(P=.001)$ and after $5 \mathrm{y}(P<.001)$, as well as in the supine position at NIV initiation $(P=.003)$ and after $5 \mathrm{y}$ $(P<.001)$.

\section{Discussion}

To date, a relatively large number of articles on the changes in quality of life, survival rates, and respiratory status after using NIV for different NMD types have been published. ${ }^{3-5,12,23-25}$ However, this is the first study in which a large number of subjects with different types of NMD were individually followed for $\geq 5 \mathrm{y}$ and whose survival and NIV use were compared. In this study, 134 of 180 subjects continued NIV for $\geq 5 \mathrm{y}$, showing a high proportion of subjects who tolerated NIV, although differences were found among various subgroups. The 5-y NIV maintenance rates were $23 \%, 89 \%$, and $91 \%$ and the average NIV maintenance durations were $21.5 \pm 19.3$ months, $55.2 \pm 11.5$ months, and $57.5 \pm 8.3$ months for groups with ALS, DMD, and SMA-congenital myopathy, respectively.

A significant difference in NIV maintenance rates between subjects with ALS and other groups seems reasonable, considering the different natural course of the disease. Subjects started on NIV who experienced additional symptoms of respiratory failure (ie, poor secretion clearance) or had progressive bulbar symptoms with ALS were changed to invasive ventilation. Unlike the other neuromuscular diseases, patients with ALS eventually need invasive mechanical ventilation due to severe progressive bulbar dysfunction. In this study, 21 subjects received a tracheostomy tube during the follow-up period, and all but 5 had ALS. In fact, Dreyer et $\mathrm{al}^{4}$ showed in a large-population cohort study that NIV followed by invasive venti- lation has a significant effect on survival in subjects with ALS. 4

In previous studies, tolerance for NIV was usually calculated by the ratio of subjects who adapted well to this mode in the initial stage. ${ }^{24,26,27}$ Farrero et $\mathrm{al}^{24}$ reported that the ratio of subjects with ALS using NIV was 50\% among those who did not have accompanying bulbar symptoms, which was higher than those of previous studies. The authors pointed out that such outcomes resulted from a multidisciplinary-team approach, the subjects' trust of providers who had extensive experience of $\geq 500$ cases, and subjects' positive attitude about the use of NIV in the in-patient setting. In our study, however, because we included only subjects who agreed to use and were able to adapt to NIV, we were not able to compare tolerance in this manner. When we defined the tolerance by the ratio of subjects maintaining NIV for $5 \mathrm{y}$, however, we concluded that a high proportion of subjects tolerated NIV. Such an outcome can probably be attributed, as in the previous study, to our multidisciplinary-team approach, our experience with $>500$ cases, and our constant management through application and adjustment of NIV settings in the in-patient setting.

Daily NIV duration increased significantly after $5 \mathrm{y}$ in the ALS group (8.0 h to $24.0 \mathrm{~h}, P<.001)$ and in the DMD group ( $8.0 \mathrm{~h}$ to $12.0 \mathrm{~h}, P<.001)$, while there was only a trend toward increased duration in the SMA-congenital myopathy group $(8.0 \mathrm{~h}$ to $9.0 \mathrm{~h}, P=.11)$. Of 40 subjects with ALS, 32 were eventually dependent on mechanical ventilation for $>23 \mathrm{~h}$; among them, 16 subjects continued with NIV.

In subjects with DMD, 18 were dependent on mechanical ventilation for $>23 \mathrm{~h}$. However, tracheostomy was rare in this group. Only 1 subject underwent tracheostomy, while the other 17 were maintained on continuous NIV. These results are similar to those from a study by Bach et al, ${ }^{18}$ in which 26 of 101 subjects with DMD who used nocturnal-only NIV became continuously dependent on NIV. In subjects with advancing DMD, continuous NIV was found to prolong life and obviate tracheostomy. 
The overall survival rate after $5 \mathrm{y}$ in our study was $79 \%$ in the total population. While the percentage of subjects who survived after 5 y was approximately $90 \%$ in those with DMD, SMA-congenital myopathy, and other NMDs, it was only $38 \%$ in subjects with ALS. However, we could not compare our survival results with long-term survival rates reported in other studies because we had a considerable number of subjects who were lost to follow-up.

Our study has several limitations. First, some subjects were lost during follow-up in each group, which resulted in a smaller sample size and increased the size differences among the groups. The other NMD group had the largest number of subjects lost during follow-up, followed by the groups with ALS and DMD, for a total drop-out rate of $28 \%$. Excluding the other NMD group, 17 of 57 subjects in the ALS group were lost, 2 of 25 subjects in the SMAcongenital myopathy group were lost, and 19 of 104 subjects were lost in the DMD group. However, because we are still providing NIV and the number of NMD cases using NIV is increasing, we expect to have additional data when we continue the follow-up observation for a longer period.

We did not collect subjective parameters, such as healthrelated quality of life. It is well known that the quality of life of patients with NMDs has improved because of the use of NIV. Data on how quality of life has actually changed for the same patients before and after using NIV for $5 \mathrm{y}$ would have been very useful. Unfortunately, because we could not consistently accumulate this information, these results were excluded.

Finally, we did not include variables other than FVC to evaluate respiratory status. Our institution also takes measures of maximal inspiratory pressure, maximal expiratory pressure, sniff nasal inspiratory pressure, and cough peak flow, in addition to FVC. FVC and cough peak flow are also measured under maximal insufflation capacity and assisted conditions. We chose FVC because this variable had the smallest data loss for the 5-y period. Khirani et al28 reported that the optimal indicators for evaluating disease progression in subjects with DMD were vital capacity and sniff nasal inspiratory pressure, which reflect the natural history of the respiratory muscle strength or decline and pulmonary function.

For subjects with ALS, Martinez et al ${ }^{19}$ used 2 physiologic indicators-vital capacity and maximum inspiratory pressure-for starting NIV, although the situation may change for these patients as bulbar symptoms progress. For subjects with SMA-congenital myopathy, unlike for subjects in the other 2 groups, mean vital capacity increased slightly after $5 \mathrm{y}$. However, we could not explain this solely by NIV use, and we could not draw any conclusions from the small sample size. Further studies with larger samples and more variables are needed.

\section{Conclusions}

With increasing medical information and government support, more patients with NMDs are using NIV. This mode has helped prolong survival and improve quality of life compared to results using invasive ventilation. Although there are various reports on the use of NIV, our study is significant because it followed subjects with diverse NMDs individually for $5 \mathrm{y}$. NIV was tolerated for long periods without increasing daily application time for most subjects with NMDs. However, those with ALS who develop severe bulbar dysfunction may be unable to continue NIV.

\section{REFERENCES}

1. Bach JR. A comparison of long-term ventilatory support alternatives from the perspective of the patient and care giver. Chest 1993;104(6): 1702-1706.

2. Ishikawa Y, Miura T, Ishikawa Y, Aoyagi T, Ogata H, Hamada S, et al. Duchenne muscular dystrophy: survival by cardio-respiratory interventions. Neuromuscul Disord 2011;21(1):47-51.

3. Villanova M, Brancalion B, Mehta AD. Duchenne muscular dystrophy: life prolongation by noninvasive ventilatory support. Am J Phys Med Rehabil 2014;93(7):595-599.

4. Dreyer P, Lorenzen CK, Schou L, Felding M. Survival in ALS with home mechanical ventilation non-invasively and invasively: a 15year cohort study in west Denmark. Amyotroph Lateral Scler Frontotemporal Degener 2014;15(1-2):62-67.

5. Bourke SC, Tomlinson M, Williams TL, Bullock RE, Shaw PJ, Gibson GJ. Effects of non-invasive ventilation on survival and quality of life in patients with amyotrophic lateral sclerosis: a randomised controlled trial. Lancet Neurol 2006;5(2):140-147.

6. Mehta S, Hill NS. Noninvasive ventilation. Am J Respir Crit Care Med 2001;163(2):540-577.

7. Make BJ, Hill NS, Goldberg AI, Bach JR, Criner GJ, Dunne PE, et al. Mechanical ventilation beyond the intensive care unit: report of a consensus conference of the American College of Chest Physicians. Chest 1998;113(5 Suppl):289s-344s.

8. Benditt JO. Full-time noninvasive ventilation: possible and desirable. Respir Care 2006;51(9):1005-1012.

9. Servera E, Sancho J, Zafra MJ, Catala A, Vergara P, Marin J. Alternatives to endotracheal intubation for patients with neuromuscular diseases. Am J Phys Med Rehabil 2005;84(11):851-857.

10. Cazzolli PA, Oppenheimer EA. Home mechanical ventilation for amyotrophic lateral sclerosis: nasal compared to tracheostomy-intermittent positive pressure ventilation. J Neurol Sci 1996;139(Suppl): 123-128.

11. Bach JR, Goncalves MR, Hon A, Ishikawa Y, De Vito EL, Prado F, et al. Changing trends in the management of end-stage neuromuscular respiratory muscle failure: recommendations of an international consensus. Am J Phys Med Rehabil 2013;92(3):267-277.

12. Bach JR. Amyotrophic lateral sclerosis: prolongation of life by noninvasive respiratory AIDS. Chest 2002;122(1):92-98.

13. Sancho J, Servera E, Banuls P, Marin J. Prolonging survival in amyotrophic lateral sclerosis: efficacy of noninvasive ventilation and uncuffed tracheostomy tubes. Am J Phys Med Rehabil 2010;89(5): 407-411.

14. Ionita C, Iannaccone ST. Treatment and management of spinal muscular atrophy and congenital myopathies. In: Bertorini TE, ed. $\mathrm{Neu}$ romuscular Disorders: Treatment and Management. Saint Louis: W. B. Saunders, 2011;179. 


\section{LONG-TERM OUTCOMES OF NIV IN NMD}

15. Han JJ, Kilmer DD. Myopathy. In: Frontera WR, ed. Delisa's Physical Medicine \& Rehabilitation. Philadelphia: Lippincott Williams \& Wilkins, 2010;773.

16. Hess DR. The growing role of noninvasive ventilation in patients requiring prolonged mechanical ventilation. Respir Care 2012;57(6): 900-918.

17. Birnkrant DJ, Bushby KM, Amin RS, Bach JR, Benditt JO, Eagle M, et al. The respiratory management of patients with duchenne muscular dystrophy: a DMD care considerations working group specialty article. Pediatr Pulmonol 2010;45(8):739-748.

18. Bach JR, Martinez D. Duchenne muscular dystrophy: continuous noninvasive ventilatory support prolongs survival. Respir Care 2011; 56(6):744-750.

19. Martinez D, Sancho J, Servera E, Marin J. Tolerance of volume control noninvasive ventilation in subjects with amyotrophic lateral sclerosis. Respir Care 2015;60(12):1765-1771.

20. Clinical indications for noninvasive positive pressure ventilation in chronic respiratory failure due to restrictive lung disease, COPD, and nocturnal hypoventilation-a consensus conference report. Chest 1999; 116(2):521-534

21. Miller RG, Jackson CE, Kasarskis EJ, England JD, Forshew D, Johnston W, et al. Practice parameter update: the care of the patient with amyotrophic lateral sclerosis: multidisciplinary care, symptom management, and cognitive/behavioral impairment (an evidence-based review): report of the Quality Standards Subcommittee of the American Academy of Neurology. Neurology 2009;73(15):1227-1233.
22. Andersen PM, Abrahams S, Borasio GD, de Carvalho M, Chio A, Van Damme P, et al. EFNS guidelines on the clinical management of amyotrophic lateral sclerosis (MALS)-revised report of an EFNS task force. Eur J Neurol 2012;19(3):360-375.

23. Chio A, Calvo A, Moglia C, Gamna F, Mattei A, Mazzini L, et al. Non-invasive ventilation in amyotrophic lateral sclerosis: a 10-year population-based study. J Neurol Neurosurg Psychiatry 2012;83(4): 377-381.

24. Farrero E, Prats E, Povedano M, Martinez-Matos JA, Manresa F, Escarrabill J. Survival in amyotrophic lateral sclerosis with home mechanical ventilation: the impact of systematic respiratory assessment and bulbar involvement. Chest 2005;127(6):2132-2138.

25. Meinesz AF, Bladder G, Goorhuis JF, Fock JM, Staal-Schreinemachers AL, Zijlstra JG, et al. [18 years experience with mechanical ventilation in patients with Duchenne muscular dystrophy]. Ned Tijdschr Geneeskd 2007;151(33):1830-1833.

26. Kleopa KA, Sherman M, Neal B, Romano GJ, Heiman-Patterson T. Bipap improves survival and rate of pulmonary function decline in patients with ALS. J Neurol Sci 1999;164(1):82-88.

27. Aboussouan LS, Khan SU, Meeker DP, Stelmach K, Mitsumoto H. Effect of noninvasive positive-pressure ventilation on survival in amyotrophic lateral sclerosis. Ann Intern Med 1997;127(6):450-453.

28. Khirani S, Ramirez A, Aubertin G, Boule M, Chemouny C, Forin V, et al. Respiratory muscle decline in Duchenne muscular dystrophy. Pediatr Pulmonol 2014;49(5):473-481.

This article is approved for Continuing Respiratory Care Education credit. For information and to obtain your CRCE

(free to AARC members) visit www.rcjournal.com

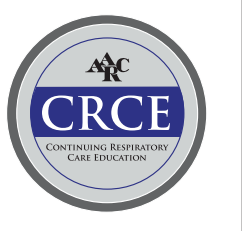

